\title{
A Critical Review of Unpowered Performance Metrics of Impedance-Type Haptic Devices
}

\author{
I. Görgülü, G. Kiper and M. I. C. Dede \\ Izmir Institute of Technology, Turkey, e-mail: ibrahimcangorgulu, gokhankiper, \\ candede@iyte.edu.tr
}

\begin{abstract}
A kinesthetic haptic device's performance relies on unpowered, powered and controlled system characteristics. In this paper, a critical review is carried out for the well-known metrics for kinematics, stiffness and dynamic aspects of robots that can be applied in evaluating the unpowered system performance of kinesthetic haptic devices. The physical meanings of these metrics are discussed and the important factors that affect the unpowered system performance of a kinesthetic haptic device are revealed.
\end{abstract}

Key words: Haptics, Performance Metrics, Stiffness, Kinematics, Dynamics

\section{Introduction}

Haptic devices are interfaces that enable the interaction of the human with a slave environment either virtual or a real one and they can be categorized with respect to their targeted touch sensation as kinesthetic and cutaneous. The devices constructed to stimulate kinesthetic sensation can be further categorized as impedance and admittance type devices. Impedance-type devices acquire the human motion and transmit this information to a slave environment while the interaction forces measured or calculated in the slave environment is displayed by these devices to the human. The quality of the haptic interaction depends on unpowered, powered and controlled system characteristics of this haptic device as explained in [16].

In [16], powered and controlled system performances were investigated in detail but, in terms of the unpowered system characteristics, some performance metrics were listed but not critically reviewed. The focus of this work is the evaluation of impedance-type haptic devices based on the unpowered system performance, which is only related with the mechanical properties of the device. The unpowered system performance is investigated in three main topics: Kinematics, Stiffness, and Dynamics, which are among the performance metrics of robot manipulators. There are some studies that investigate these metrics with case studies [4] however, this work reviews these metrics and relates them to the haptic device performance metrics. 
In the optimal design of impedance-type haptic device manipulators, the desired objective is to minimize the minimum mechanical impedance, maximize the maximum mechanical impedance and enlarge the operational frequency range, the bandwidth. Minimum impedance is achieved when the back-drivability is at its best conditions, which require the minimization of dynamic effects by constructing a lightweight manipulator and by maximizing the manipulability. Maximum impedance, on the other hand, can be achieved when the manipulator is the least back-drivable, which states that the requirements of the minimum impedance are reversed. There are also other critical constraints in the design optimization of a haptic device such as workspace and isotropy of the force and velocity ellipsoids. The objective function construction becomes highly nonlinear due to the competitive relationship between these constraints and performance metrics. Therefore, an optimization algorithm such as a genetic algorithm can be used to solve the optimization problem. However, the critical issue in this solution is the composition of the objective function in a meaningful way. This work undertakes this challenge.

Manipulator kinematics is the backbone of the design procedure since the dimensions of the links affect the kinematic, stiffness and dynamic properties. On the other hand, mechanical impedance characteristics of a device are driven by its stiffness and dynamics properties. In order to understand the relation between the kinematics and impedance, all the design parameters affecting the impedance performance should be considered simultaneously. Therefore, the next sections are organized to review the kinematics, stiffness, and dynamics related performance metrics and finally conclude the paper by providing a discussion on the adaptation of these metrics in the optimal design of haptic device manipulators.

\section{Kinematics Performance Metrics}

Kinematic performance of the manipulators is usually evaluated depending on their dexterity measure. Dexterity is evaluated in terms of manipulability and condition number. Both metrics use the Jacobian matrix but evaluate its different properties. Thus, as a first step, Jacobian matrix properties should be understood.

The Jacobian matrix is the mapping matrix of the velocities between the joint space and task space in which its elements are called the velocity influence coefficients. Eigenvalues and eigenvectors of the Jacobian matrix reveal the physical meaning of Jacobian matrix for a unit change in joint space velocities [6]. Eigenvectors of the Jacobian matrix of a 3 degree of freedom ( $\mathrm{DoF}$ ) translational manipulator, for instance, represent the orientation of an ellipsoid's axes for a specific pose of the manipulator. Eigenvalues of the matrix define the semi-axis dimensions of this ellipsoid. This ellipsoid is named as velocity ellipsoid and the boundary of the ellipsoid shows the motion capability of the manipulator in an arbitrary direction.

Higher eigenvalues correspond to higher motion capability which is desired for minimizing the minimum impedance of a haptic device. The manipulability concept is introduced in [18] to describe the easiness of motion of the end effector. Here, the 
determinant of the Jacobian is used as the performance measure. The manipulability measure is shown in Eq. (1) for both redundant and non-redundant manipulators.

$$
\mu_{v}=\sqrt{\operatorname{det}\left(\hat{J} \hat{J}^{T}\right)}
$$

Here, $\hat{J}$ is the Jacobian matrix, $\mu_{v}$ is the manipulability measure. This metric is a value that is proportional to the volume of the velocity ellipsoid. If the Jacobian matrix is square, the absolute value of its determinant is used for evaluation [12].

Condition number calculates the magnitute of ratio between the maximum and minimum eigenvalues of Jacobian matrix. The ratio is evaluated in order to understand the motion resolution of the mechanism [15]. The formulation is shown as:

$$
c_{v}=\|\hat{J}\|\left\|\hat{J}^{-1}\right\|,
$$

where $c_{v}$ is the condition number calculated by using the Euclidean norm of the Jacobian matrix and its inverse. If Jacobian matrix is a non square matrix, psuedo inverse can be used. The resolution of the acquired motion is important in haptic devices since the acquired motion is the demand for the slave system that might be employed in a critical-precision operation such as telesurgery.

A key activity of a haptic device is reflecting forces back to the user. Therefore, in contrast to the previous motion-related metrics, force-related metrics should be evaluated. The relation between the actuator inputs, $\bar{\tau}$, and the end-effector forcing, $\bar{F}$, is established by using the Jacobian matrix as shown in Eq.(3).

$$
\bar{F}=\left(\hat{J}^{-1}\right)^{T} \bar{\tau}
$$

The force capability, $\mu_{f}$, of the manipulator can be written as a scalar index as:

$$
\mu_{f}=\sqrt{\operatorname{det}\left(\hat{J} \hat{J}^{T}\right)^{-1}}=1 / \mu_{v} .
$$

In haptic device design, it is favorable to maximize the force capability, which results in receiving the most force output for unit input to the actuators. This would maximize the maximum impedance of the device. On the other hand, maximization of this metric means the minimization of the manipulability. Therefore, minimizing the minimum impedance and maximizing the maximum impedance by using these metrics are contradictory goals and a trade-off should be adjusted by the designer.

The abovementioned metrics are generalized and normalized for a fair comparison between different manipulators. If the design problem of the manipulator consists of the selection of manipulators which have different degree-of-freedom (DoF), the comparison between the performance metrics will be impossible since they have different physical meaning (area, volume). This order dependency can be solved with the manipulation in Eq.(1) and Eq.(4) as proposed in [11], which is :

$$
\mu_{v}^{n}=\sqrt[n]{\operatorname{det}\left(\hat{J} \hat{J}^{T}\right)} \text { and } \mu_{f}^{n}=\sqrt[n]{\operatorname{det}\left(\hat{J} \hat{J}^{T}\right)^{-1}}=1 / \mu_{v}^{n}
$$


where $n$ is the rank of the Jacobian matrix (instantaneous DoF of the manipulator).

Another problem stated in [11] is the scaling problem. In order to evaluate the different size manipulators in a common framework, the characteristic length is introduced in [1]. This length is used to normalize the Jacobian matrix. The characteristic length $L$ is defined as the ratio between the maximum desired reach, $R_{d}$, and the maximum actual reach, $R_{a}$, which can be achieved by synthesized links. For a normalized Jacobian matrix, $\hat{J}_{n}$, the desired reach is 1 .

$$
\hat{J}_{n}=\hat{J} L \text { for } R_{d}=1, \text { where } L=\frac{R_{d}}{R_{a}}
$$

Another problem in using kinematic performance indices arises if the manipulator has both translational and rotational DoFs. In this case, Eqs.(1) and (3) lose their physical meanings. A solution is devised by using homogeneous coordinates [10].

\section{Stiffness Performance Metrics}

The stiffness of a haptic device manipulator determines the maximum impedance along with the damping and inertial properties of the system. This property directly determines the quality and the limits of rendered forces in a haptics scenario. The stiffness of the manipulator can be analyzed using finite element methods (FEM).

Stiffness matrix can be obtained by using Hooke's law [7] or virtual work method [14]. In Eq.(7), the most general form of force-stiffness relation is presented where $\hat{K}_{C}$ is the Cartesian stiffness matrix, and $\delta \bar{r}$ denotes the deflection at the tip point. Eq.(8) represents the joint reactions $\left(\bar{F}_{\theta}\right)$-task space force relation and Eq.(9) represents the joint reaction-joint space deflections $(\delta \bar{\theta})$ relation. Here, $\hat{K}_{\theta}$ denotes the structural stiffness of the link of interest.

$$
\begin{gathered}
\bar{F}=\hat{K}_{C} \delta \bar{r} \\
\bar{F}_{\theta}=\hat{J}_{\theta}^{T} \bar{F} \\
\bar{F}_{\theta}=\hat{K}_{\theta} \delta \bar{\theta}
\end{gathered}
$$

Using the Conservative Congruency Transformation [5], the mapping between the joint space stiffness matrix $\hat{K}_{\theta}$ and Cartesian space stiffness matrix is as follows:

$$
\hat{K}_{C}=\left(\hat{J}_{\theta} \hat{K}_{\theta}^{-1} \hat{J}_{\theta}^{T}\right)^{-1}
$$

where the $\hat{J}_{\theta}$ denotes the homogeneous Jacobian matrix developed for virtual joint variables. Here, $\hat{K}_{\theta}$ can be a diagonal matrix for a simplified model approach or nondiagonal matrix which represents the real case. Cartesian space stiffness is critical in design since it directly relates the external forces and tip point deflections.

Kinematic evaluation methods can be extended for stiffness matrix [3]. Singular value decomposition (SVD) of $\hat{K}_{C}$ reveals directional stiffness properties of the ma- 
nipulator. Similar to force and velocity ellipsoids, SVD can be used for graphical illustration of stiffness. Frobenius norm of $\hat{K}_{C}$ is used to evaluate overall stiffness of the manipulator, $S_{f}$, ib Eq.(11). On the other hand, the Euclidean norm of $\hat{K}_{C}$ and $\left(\hat{K}_{C}\right)^{-1}$ denoted by $S_{e}$ exposes the stiffest and the most compliant axes of the manipulator which is similar to condition number, Eq.(11).

$$
S_{f}=\sqrt{\operatorname{tr}\left(\hat{K}_{C} \hat{K}_{C}^{T}\right)} \text { and } S_{e}=\left\|\hat{K}_{C}\right\|\left\|\left(\hat{K}_{C}\right)^{-1}\right\|
$$

If $S_{e}$ value is equal to 1, it can be stated that the manipulator is in an isotropic pose in terms of stiffness. This index is useful when the link weights and dynamic effects are included to the calculation of stiffness matrix. Hence, manipulator can be designed to be more stiff along the axes affected by gravitational and dynamic forces.

Another scalar index can be defined as the determinant of $\hat{K}_{C}$ shown in Eq.(12). $S_{d}$ is a value that is proportional to the the volume of stiffness ellipsoid. Naturally, higher volume indicates higher stiffness.

$$
S_{d}=\operatorname{det}\left(\hat{K}_{C}\right)=\operatorname{det}\left(\hat{J}_{\theta} \hat{K}_{\theta}^{-1} \hat{J}_{\theta}^{T}\right)^{-1}
$$

Notice that, $S_{d}$ can be increased by increasing the determinant of $\hat{K}_{\theta}$ and/or by decreasing determinant of $\hat{J}_{\theta}$. If the manipulator is in a singular pose $S_{d}$ becomes infinite. Therefore, the designer should be careful while using stiffness oriented performance metrics as a design objective and should make use of global indices to resolve this problem. If the design parameters consist of link lengths, which is the general case, in order to avoid such problems, kinematic synthesis should be concluded before designing the link geometry for desired stiffness.

\section{Dynamics Performance Metrics}

Dynamic properties are hard to implement to design procedure since, dimensional parameters must be solved first. Therefore, dynamics oriented designs are iterative. Acceleration mapping between the task and joint space in Eq.(13) and dynamic equation of motion in Eq.(14) are manipulated to define $\ddot{\vec{r}}^{*}$ and $\bar{\tau}^{*}$, respectively.

$$
\begin{gathered}
\ddot{\bar{r}}=\hat{J} \ddot{\bar{\theta}}+\dot{\hat{J}} \dot{\bar{\theta}} \Rightarrow \ddot{r}^{*}=\ddot{\vec{r}}-\dot{\hat{J}} \dot{\bar{\theta}} \\
\bar{\tau}=\hat{M} \ddot{\bar{\theta}}+\hat{V} \dot{\bar{\theta}} \Rightarrow \bar{\tau}^{*}=\bar{\tau}-\hat{V} \dot{\bar{\theta}}
\end{gathered}
$$

where $\bar{\tau}$ contains the actuator inputs, $\hat{M}$ is the generalized inertia matrix, $\hat{V}$ contains the Coriolis and centripetal effects and $\bar{\theta}$ is the vector of generalized coordinates (joint variables). Further manipulation can be issued to transform the Eq.(14) from joint space to Cartesian space by using Eq.(3) and Eq.(15) can be obtained.

$$
\bar{F}^{*}=\hat{M}_{C} \ddot{\bar{r}}^{*} \text { where } \hat{M}_{C}=\hat{J}^{-T} \hat{M} \hat{J}^{-1}
$$


Asada referred to the $\hat{M}_{C}$ matrix as generalized inertia matrix (GIM), however, this GIM is derived by using the generalized coordinates as Cartesian space pose components [2]. He used the eigenvalues and eigenvectors in order to graphically illustrate the properties of GIM and named them as generalized inertia ellipsoids (GIE). In terms of haptics, $\hat{M}_{C}$ is the dominating factor of minimum impedance. If a user jiggles the end effector with $\ddot{r}^{*}$ input, the felt force due to the dynamic effects is the $\bar{F}^{*}$ and the relationship between the input and output is established by the $\hat{M}_{C}$ matrix. Therefore, for minimization of the minimum impedance of a haptic device, the $\hat{M}_{C}$ matrix should be minimized.

Similar evaluations conducted for stiffness performance can be repeated for GIM. Average inertia of the manipulator, $I_{f}$, and inertia condition number, $I_{e}$, as the Euclidean norm of inertia matrix are defined in Eq.(16).

$$
I_{f}=\sqrt{\operatorname{tr}\left(\hat{M}_{C} \hat{M}_{C}^{T}\right)} \text { and } I_{e}=\left\|\hat{M}_{C}\right\|\left\|\left(\hat{M}_{C}\right)^{-1}\right\|
$$

By using Eq.(13) and Eq.(14), Eq.(17) is derived. By using this, in [17], the dynamic manipulability measure is introduced (Eq.(17)). This index indicates the amplification rate between the actuator inputs and output acceleration of the endeffector. Better dynamic manipulability can be achieved by increasing the manipulability and/or decreasing the determinant of inertia matrix.

$$
I_{d}=\operatorname{det}\left(\hat{J} \hat{M}^{-1}\right)=\operatorname{det}(\hat{J}) / \operatorname{det}(\hat{M})
$$

Another evaluation approach in [9] proposed the acceleration radius measure to emphasize the acceleration capability of the end-effector for any arbitrary direction. However, $I_{e}$ and $I_{d}$ are more suitable as performance indices since they already consider the acceleration radius.

Final evaluation can be carried out by computing the natural frequency of the manipulator. Natural frequency can be computed by evaluating the eigenvalues of the dynamic matrix $\hat{D}$ which is defined in Eq.(18) in joint space and in Cartesian space. Modal vectors are the eigenvectors of the dynamic matrix.

$$
\hat{D}=-\hat{M}^{-1} \hat{K} \text { and } \hat{D}_{C}=\hat{J} \hat{D} \hat{J}^{-1}
$$

Increasing the natural frequency corresponds to increasing bandwidth and decreasing the response time of the mechanism. This also maximizes the maximum impedance by increasing gains of $\hat{K}$ and minimizes the minimum impedance by decreasing gains of $\hat{M}$. It should be noted that $\hat{K}_{C}$ and $\hat{M}_{C}$ are functions of link lengths and joint variables. Thus, natural frequency can be intuitively optimized in kinematic level. Matrix $\hat{D}$ or $\hat{D}_{C}$ should be simplified as a scalar performance index. The easiest way is by evaluating the Frobenius norm of the dynamic matrix (Eq.(19)), which takes in account the value of natural frequency for Cartesian space, $\omega_{n c}$, and for joint space, $\omega_{n}$. This way an average value of natural frequency is obtained.

$$
\omega_{n c}=\sqrt{\operatorname{tr}\left(\hat{D}_{C} \hat{D}_{C}^{T}\right)} \text { and } \omega_{n}=\sqrt{\operatorname{tr}\left(\hat{D} \hat{D}^{T}\right)}
$$




\section{Globalization of Indicies}

All indices, mentioned above, are pose depended. A manipulator cannot be designed for a single pose. It should be evaluated at each discrete pose. The global performance index is proposed by Gosselin and Angeles [8] to address this problem:

$$
k_{i}=\left(\int_{W} i d w\right) /\left(\int_{W} d w\right),
$$

where $k_{i}$ is the average of the globalized index, $i$ is the index which will be globalized and $W$ denotes the workspace. As a scalar value, $k_{i}$ enables evaluating the performance of related index for all discrete positions of the workspace. If there are no singular poses within the workspace, this method can be simplified by considering only the critical poses which are generally at the boundaries of the workspace.

Evaluation of $k_{i}$ alone may mislead the designer. If the manipulator is close to a singular pose, the effect of the index at that pose will be reduced when the average value is calculated. In [13] the uniformity of the performance index is introduced as

$$
U=I_{\min } / I_{\max }
$$

where $U$ is the uniformity measure, $I_{\min }$ and $I_{\max }$ are the minimum and maximum values of the observed index through the workspace. As an example, the uniformity of the force capability is especially important for haptic devices so that same amount of impedance can be displayed to the user throughout the workspace.

\section{Conclusions}

The critical metrics to optimize the unpowered system characteristics of a haptic device are reviewed and discussed in terms of kinematics, stiffness and dynamics indices, and their physical meanings. All indices include the Jacobian matrix or share the same parameters with the Jacobian matrix. Therefore, these metrics can be narrowed down as a function of Jacobian matrix entries and it is possible to optimize all performance indices in kinematic level up to a certain point. This is computationally faster and it handles most of the design parameters, simultaneously. However, Jacobian matrices should be normalized and made homogeneous to remove the scale and unit dependency to compare different manipulator topologies in the same framework. The metrics investigated in this paper will be applied in the optimal design of a new haptic device.

Acknowledgements This work is supported in part by The Scientific and Technological Research Council of Turkey via grant number 117M405. 


\section{References}

1. Angeles, J.: Fundamentals of robotic mechanical systems, vol. 2. Springer (2002)

2. Asada, H.: A geometrical representation of manipulator dynamics and its application to arm design. Journal of dynamic systems, measurement, and control 105(3), 131-142 (1983)

3. Carbone, G., Ceccarelli, M.: Comparison of indices for stiffness performance evaluation. Frontiers of Mechanical Engineering in China 5(3), 270-278 (2010)

4. Ceccarelli, M., Carbone, G., Ottaviano, E.: Multi criteria optimum design of manipulators. Bulletin of the polish academy of sciences technical sciences $\mathbf{5 3}$ (2005)

5. Chen, S.F., Kao, I.: Conservative congruence transformation for joint and cartesian stiffness matrices of robotic hands and fingers. The International Journal of Robotics Research 19(9), 835-847 (2000)

6. Chiu, S.L.: Kinematic characterization of manipulators: an approach to defining optimality. In: Robotics and Automation, 1988. Proceedings., 1988 IEEE International Conference on, pp. 828-833. IEEE

7. Gosselin, C.: Stiffness mapping for parallel manipulators. IEEE Transactions on Robotics and Automation 6(3), 377-382 (1990)

8. Gosselin, C., Angeles, J.: A global performance index for the kinematic optimization of robotic manipulators. Journal of Mechanical Design 113(3), 220-226 (1991)

9. Graettinger, T.J., Krogh, B.H.: The acceleration radius: a global performance measure for robotic manipulators. IEEE Journal on Robotics and Automation 4(1), 60-69 (1988)

10. Khan, W.A., Angeles, J.: The kinetostatic optimization of robotic manipulators: the inverse and the direct problems. Journal of mechanical design 128(1), 168-178 (2006)

11. Kim, J.O., Khosla, K.: Dexterity measures for design and control of manipulators. In: Intelligent Robots and Systems' 91.'Intelligence for Mechanical Systems, Proceedings IROS'91. IEEE/RSJ International Workshop on, pp. 758-763. IEEE (1991)

12. Paul, R.P., Stevenson, C.N.: Kinematics of robot wrists. The International journal of robotics research 2(1), 31-38 (1983)

13. Pham, H.H., Chen, I.M.: Optimal synthesis for workspace and manipulability of parallel flexure mechanism. In: 11th World Congress in Mechanism and Machine Science, Tianjin, China, Aug, pp. 18-21 (2003)

14. Quennouelle, C., Gosselin, C.: Stiffness matrix of compliant parallel mehanisms. In: ASME 2008 International Design Engineering Technical Conferences and Computers and Information in Engineering Conference, pp. 151-161. American Society of Mechanical Engineers

15. Salisbury, J.K., Craig, J.J.: Articulated hands: Force control and kinematic issues. The International journal of Robotics research 1(1), 4-17 (1982)

16. Samur, E.: Performance metrics for haptic interfaces. Springer Science \& Business Media (2012)

17. Yoshikawa, T.: Dynamic manipulability of robot manipulators. Transactions of the Society of Instrument and Control Engineers 21(9), 970-975 (1985)

18. Yoshikawa, T.: Manipulability of robotic mechanisms. The international journal of Robotics Research 4(2), 3-9 (1985) 